\title{
Visible to the Eye
}

Was ist das Schwerste von allem?

Was dir das Leichteste dünket:

Mit den Augen zu sehen,

Was vor den Augen dir liegt.

GOETHE

Diese Verse stellte Walter Sandritter seinem Lehrbuch und Atlas der Histopathologie voran. Viele Generationen von Medizinstudenten werden sich noch an Sandritters Histopathologie und Makropathologie erinnern, die das Bild, den optischen Eindruck, in den Vordergrund der „Belehrung“ rückten. Der Verfasser dieses Editorials hatte persönlich das Glück, als Medizinstudent in Kursen und Vorlesungen und später als wissenschaftlicher Assistent im Sektionssaal des Pathologischen Instituts „Ludwig Aschoff-Haus“ der Universität Freiburg das didaktische Geschick Sandritters erleben zu dürfen.

Bewusstes Sehen, die richtige Interpretation des Geschehenen und schließlich die Zusammenfassung der optischen Eindrücke zu einer „Synopsis“ sind wichtige Voraussetzungen für erfolgreiches ärztliches Diagnostizieren. Um klinische Blickdiagnosen stellen zu können, muss man vorher wissen, wonach man Ausschau halten soll. Mit den Augen erkennen wir besondere klinische Merkmale des Patienten, betrachten Röntgen- und CT-Bilder, schauen durchs Bronchoskop und auf den Operationssitus, blicken ins Mikroskop, auf Hauttestergebnisse, Schlaflaborkurven und Resistanceschleifen - pneumologische Blickdiagnosen sind also in vielen Bereichen möglich.

In der neuen Rubrik „Pneumologische Blickdiagnose" ( siehe Seite 369) steht das Bild im Mittelpunkt. Der Begleittext soll in knapper Form Anamnese, Befunde und Verlauf schildern, Kernaussagen zum dargestellten Krankheitsbild treffen und daraus resultierende Schlussfolgerungen erläutern. Der Umfang sollte eine Druckseite nicht überschreiten (2-3 Abbildungen und maximal 1 Manuskriptseite Text). Ich möchte an dieser Stelle dazu einladen, derartige Beiträge als „Blickdiagnosen“ einzureichen. Lehren wir uns gegenseitig das Schwerste von allem: „Mit den Augen zu sehen, was vor den Augen uns liegt.“

Ulrich Costabel, Essen 